\title{
Psychologische Interventionen wirksamer als Standardtherapie
}

Fragestellung: Wie erfolgreich ist die achtsamkeitsbasierte Stressreduktion („mindfulness-based stress reduction“, MBSR) im Vergleich zur kognitiven Verhaltenstherapie und zur konventionellen Therapie bei Patienten mit chronischen Rückenschmerzen?

Hintergrund: Bei vielen Patienten mit chronischen Rückenschmerzen ist eine rein medikamentöse Therapie nicht ausreichend wirksam. Neben Krankengymnastik und Physiotherapie ist zumindest in Deutschland die kognitive Verhaltenstherapie fester Bestandteil der nicht medikamentösen Therapie. Bei der achtsamkeitsbasierten Stressreduktion handelt es sich um eine Gruppentherapie mit Meditation, Konzentrations- und Bewegungsübungen, wobei diese Technik in den 1970er-Jahren in den USA entwickelt wurde. Bisher gab es keine randomisierte Studie, in der die MBSR mit der kognitiven Verhaltenstherapie verglichen wurde.

Patienten und Methodik: Es handelt sich um eine randomisierte Studie an 342 Erwachsenen im Alter zwischen 20 und 70 Jahren, die in drei Therapiegruppen randomisiert wurden.

In Gruppe 1 wurde eine MBSR im Gruppensetting durchgeführt, wobei die einzelnen Sitzungen aufgezeichnet und den Teilnehmern zur Verfügung gestellt wurden.

Die Patienten der Gruppe 2 erhielten eine kognitive Verhaltenstherapie. Im Rahmen dieser Therapie werden die Patienten über chronische Schmerzen und den Zusammenhang zwischen Psyche, Emotionen und Co-

Cherkin DC, Sherman KJ, Balderson $\mathrm{BH}$ et al. Effect of mindfulness-based stress reduction vs cognitive behavioral therapy or usual care on back pain and functional limitations in adults with chronic low back pain: a randomized clinical trial. JAMA 2016; 315: 1240 -9 ping-Strategien aufgeklärt.

Die Patienten der dritten Gruppe wurden mittels Standardtherapie behandelt. Dabei waren sie frei, sich eine beliebige Therapieform auszusuchen.

Die Befragung der Patienten im Rahmen der Nachun- tersuchung erfolgte durch Personen, die nicht über die Zuteilung zu einer bestimmten Therapiegruppe informiert waren. Diese Interviews wurden nach vier, acht, 26 und 52 Wochen geführt. Der primäre Endpunkt wurde nach 26 Wochen erhoben. Es gab zwei primäre Endpunkte: zum einen die mithilfe des Roland Disability Questionnaires“ (Score von 0 bis 23 Punkten) erfasste Beeinträchtigung und zum anderen die selbstberichtete Rückenschmerzbeeinträchtigung auf einer Skala von 0 bis 10 .

Ergebnisse: Insgesamt wurden 342 Patienten in die Studie aufgenommen. Das mittlere Alter betrug 49 Jahre. $66 \%$ der Studienteilnehmer waren Frauen und die mittlere Dauer der Rückenschmerzen betrug 7,3 Jahre. 294 Teilnehmer, entsprechend $86 \%$, schlossen die Studie nach 26 Wochen ab und insgesamt 290 Patienten beendeten die Studie nach 52 Wochen.

Eine Verbesserung von $30 \%$ und mehr beim Roland Disability Questionnaire erreichten 60,5\% mit der MBSR, 58\% mit der kognitiven Verhaltenstherapie und $44 \%$ mit der Standardtherapie. Dieser Unterschied war bezüglich der nicht medikamentösen Therapie gegenüber der Standardtherapie signifikant. Zwischen den beiden psychologischen Therapien ergaben sich keine Unterschiede. Der prozentuale Anteil der Patienten, die eine klinisch bedeutsame Verbesserung der Schmerzen nach 26 Wochen aufwiesen, betrug $44 \%$ in der Gruppe mit MBSR, $45 \%$ in der kognitiven Verhaltens- und $27 \%$ in der Standardtherapiegruppe. Diese Unterschiede waren ebenfalls signifikant. Die Ergebnisse ließen sich auch nach 52 Wochen noch nachweisen.

Schlussfolgerungen: Die Studie zeigt, dass bei Erwachsenen mit chronischen Rückenschmerzen eine Verhaltenstherapie mit MBSR oder kognitiver Verhaltenstherapie deutlich wirksamer ist als eine medikamentöse Standardtherapie. Die Therapieerfolge sind nach 26 und 52 Wochen nachweisbar.

\section{- Kommentar von Hans-Christoph Diener, Essen}

\section{Lang anhaltende Behandlungseffekte}

Das ist die erste große randomisierte Studie bei Patienten mit chronischen Rückenschmerzen. Sie zeigt, dass beide verhaltenspsychologischen Ansätze, nämlich die MBSR und die kognitive Verhaltenstherapie, bei erwachsenen Patienten mit chronischen Rückenschmerzen wirksam sind. Das ist insbesondere aufgrund der langen Erkrankungsdauer bemerkenswert. Die chronischen Rückenschmerzen bestanden im Mittel bereits seit sieben Jahren. Ebenfalls bemerkenswert ist die Tat- sache, dass es sich hierbei nicht um einen kurzfristigen Therapieeffekt handelte, sondern dass die Wirksamkeit der Behandlungen über 26 bis 52 Wochen nachgewiesen werden konnte. Insbesondere ist wichtig, dass diese Behandlungen keinerlei Nebenwirkung haben. Leider gibt es in Deutschland nur sehr wenige Institutionen, die diese beiden nicht medikamentösen Behandlungsformen in einem Gruppen-Setting anbieten können. 